
Faculty of Music

\title{
Effect of tone sandhi on singing in Chaozhou dialect
}

\section{Introduction}

- Chaozhou dialect, a branch of Southern Min Chinese, is a tone language with eight tones and a wealth of tone sandhi (see Table 1 on the right). The tone sandhi patterns are final-prominent, the syllable in the final position of a tone sandhi group keeping the value of its citation tone while others in the non-final position undergo sandhi.

- With songs that are sung in the Chaozhou dialect the question arises of whether there is a tone-sandhi effect on melodic construction and tonal realisation. This paper aims to present empirical results from a corpus analysis of Chaozhou songs and an observational study on Chaozhou singers' realisation of tones.

\section{Corpus analysis}

- Hypothesis. Tones undergo sandhi in Chaozhou songs.

- Materials. 10 folk songs and 10 contemporary songs were included in the corpus of Chaozhou songs.

Method. We looked at the relationship between consecutive tones and consecutive notes in Chaozhou songs using two datasets: one of them was coded based on the values of citation tones (Citation dataset) and another was coded based on the values of tones when they are realised in the context with sandhi (Sandhi dataset). We conducted Pearson correlation coefficient test to assess the correlation between the pitch transitions of tones and of notes in two datasets. We also calculated the rate of matches and mismatches between two consecutive tones and two consecutive notes, taking the form of similar motion, oblique motion or contrary motion (Ladd \& Kirby, 2020).

- Result. Pearson's $r$ shows that tones and notes in Chaozhou songs were strongly correlated in Sandhi dataset, while the correlation between the two variables in Citation dataset was weak (see Table 2). There is a strikingly higher rate of similar motions in Sandhi dataset than that in Citation dataset (see Table 3).

Discussion. These outcomes show that there is a clear effect of tone sandhi on the tone-melody correspondence in Chaozhou songs.

\begin{tabular}{|l|r|r|}
\multicolumn{1}{|c|}{ Genre of songs } & Dataset & Pearson's $r$ \\
\hline Folk songs (n-1432) & Sandhi & 0.87 \\
\hline Contemporary songs $(\mathrm{n}=815)$ & Citation & 0.22 \\
& Sandhi & 0.79 \\
\hline
\end{tabular}

Table 2. The outcomes of Pearson's correlation coefficient test between tones and notes in two genres of Chaozhou songs in Sandhi dataset and Citation dataset.

\begin{tabular}{|l|l|r|r|}
\hline \multicolumn{1}{|c|}{ Genre of songs } & \multicolumn{1}{|c|}{ Motion } & Sandhi dataset & Citation dataset \\
\hline Folk songs & Similar & $\mathbf{1 2 7 1}(\mathbf{8 9 \%})$ & $621(43 \%)$ \\
& Oblique & $147(10 \%)$ & $626(44 \%)$ \\
\cline { 2 - 3 } & Contrary & $14(1 \%)$ & $185(13 \%)$ \\
\hline \multirow{2}{*}{$\begin{array}{l}\text { Contemporary } \\
\text { songs }\end{array}$} & Similar & $\mathbf{6 3 7}(\mathbf{7 8 \%})$ & $381(47 \%)$ \\
& Oblique & $150(19 \%)$ & $370(45 \%)$ \\
& Contrary & $28(3 \%)$ & $65(8 \%)$ \\
\hline
\end{tabular}

Table 2. Different degrees of tone-melody correspondence in two genres of Chaozhou songs in Sandhi dataset and Cation dataset, measured by the number of matches and mismatches. These matches are called similar motions; the mismatches, on the other hand, are divided into oblique motions and contrary motions. In similar motion, the line of tones and the line of notes; in oblique motion, one line goes up or down while the other stays at the same pitch; in contrary motion, two lines go in opposite directions.

\begin{tabular}{r|r|r|r|r|r} 
Surface Tone & Form of tone & $\mathbf{N}$ & Median & Ior & \\
\hline tone/35/ & Citation & 136 & 2.82 & 1.68 & Table 4. Samples, \\
& Sandhi & 102 & 2.97 & 1.54 & Medians and \\
\hline tone/53/ & Citation & 68 & -4.00 & 4.3 & range of four \\
& Sandhi & 68 & -1.99 & 2.33 & surface tones \\
\hline tone/213/ & Citation & 34 & 2.00 & 2.22 & $/ 35 /, / 53 /, / 213 /$ \\
& Sandhi & 136 & 1.18 & 3.32 & and $/ 21 /$ in \\
\hline tone/21/ & Citation & 102 & -0.90 & 2.84 & citation form and \\
& Sandhi & 68 & 0.97 & 3.11 & sandhi form. \\
\hline
\end{tabular}

\section{Bibliography}

Ladd, D. R., \& Kirby, J. P. (2020). Tone-melody Matching in Tone Language Singing. In C. Gussenhoven \& A. Chen (Eds.), Oxford Handbook of Linguistic Prosody (pp. 676-687).

Xu, Y. (2013). ProsodyPro - A Tool for Large-scale Systematic Prosody Analysis. In Proceedings of

\begin{tabular}{|c|c|c|c|c|c|}
\hline Tone & $\begin{array}{l}\text { Traditional } \\
\text { Names of tones }\end{array}$ & $\begin{array}{l}\text { Value } \\
\text { (citation) }\end{array}$ & $\begin{array}{l}\text { Value } \\
\text { (sandhi) }\end{array}$ & Example & $\begin{array}{l}\text { Table } 1 \text {. The citation tones } \\
\text { and tone sandhi in }\end{array}$ \\
\hline $1 \mathrm{a}$ & Yin Ping 阴平 & 33 & 23 & si - 诗poet & by using the numerical \\
\hline $1 b$ & Yang Ping 阳平 & 55 & 213 & si - 死death & representation of Chao's \\
\hline $2 a$ & Yin Shang 阴上 & $53 / 21$ & $24 / 35$ & $\mathrm{si}-$ 四four & $\begin{array}{l}\text { The variety } \\
\text { are is from the }\end{array}$ \\
\hline $2 b$ & Yang Shang 阳上 & 35 & 21 & $\mathrm{si}$ - 薛asurname "si?" & area of Chaozhou City. The \\
\hline $3 a$ & Yin Qu 阴去 & 213 & $42 / 53$ & si - 时time & underlined ' 21 ', ' 33 ' and \\
\hline $3 b$ & Yang Qu 阳去 & 11 & 12 & si - 是yes & $\begin{array}{l}\text { '54' are called Chinese } \\
\text { enterino tones or checked }\end{array}$ \\
\hline $4 a$ & Yin Ru 阴入 & $\underline{21}$ & $\underline{33} / \underline{54}$ & si - 逝disappeared & tones, being shorter in \\
\hline $4 b$ & Yang Ru 阳入 & $\underline{54}$ & 21 & si - 蚀erosion & $\begin{array}{l}\text { duration than the other } \\
\text { tones. . }\end{array}$ \\
\hline
\end{tabular}

\section{Observational study}

- Hypothesis. Tones that can be expressed in citation form and in sandhi form are realized differently when they are sung.

- Material. We used a Chaozhou song ‘Oy $a$ oy' (拥啊拥, 'I am tucking you in, my little baby') based on a traditional Chaozhou nursery rhyme. Four tones /35/, /53/, /213/ and /21/ that have the two underlying sources were included in the observational study (see Table 1 for the tones in citation form and in sandhi form, such as tone /35/, with citation $2 b$ and sandhi 2a).

- Participants. 34 Participants who are native Chaozhou speakers were invited to sing the song 'Oy a oy'.

- Method. We textgrided syllables with tones /35/, /53/, /213/ and /21/ in 34 song recordings, normalised the duration of sung syllables and extracted the values of $f 0$ at 10 equal-time points $(\mathrm{Xu}, 2015)$. We then calculated the data point that reflected the pitch change by $\Delta \mathrm{F} 0=$ fmax-fmin, where the fmax was the maximum value of the 10 time points from $f 1$ to $f 10$ and the fmin the minimum. When the fmax occurred before the fmin, the direction of pitch change was counted as fall and marked with a minus sign '-'; conversely, it was counted as rising in the opposite case. 714 data points were then measured in semitones and divided into two groups, citation and sandhi (see Table 4 on the bottom of the left).

- Results. There were highly significant differences between two underlying forms in relation to the tones $/ 53 /(\mathrm{p}<0.001)$ and $/ 21 /(\mathrm{p}<0.0001)$. However, tones $/ 35 /$ and $/ 213 /$ were performed similarly in the two forms (see Figure 1 below).

- Discussion. The significant differences may be related to the position of the syllable in a phrase that serves as a tone sandhi group. Results suggest that falling citation tones that were in the final position of a phrase tended to exhibit a larger falling contour. However, the pitch change of sandhi tones that are in non-final position may be more affected by the pitches of tones that precede or follow them. Although no significant difference was found between citation /213/ and sandhi /213/, the median pitch changes of the two forms suggested citation tones in the final position tended to be freer of expressiveness, while sandhi tones were relatively less flexible (see Table 4). Overall, the result implies a sandhi effect on the tonal realisation when tones are sung.

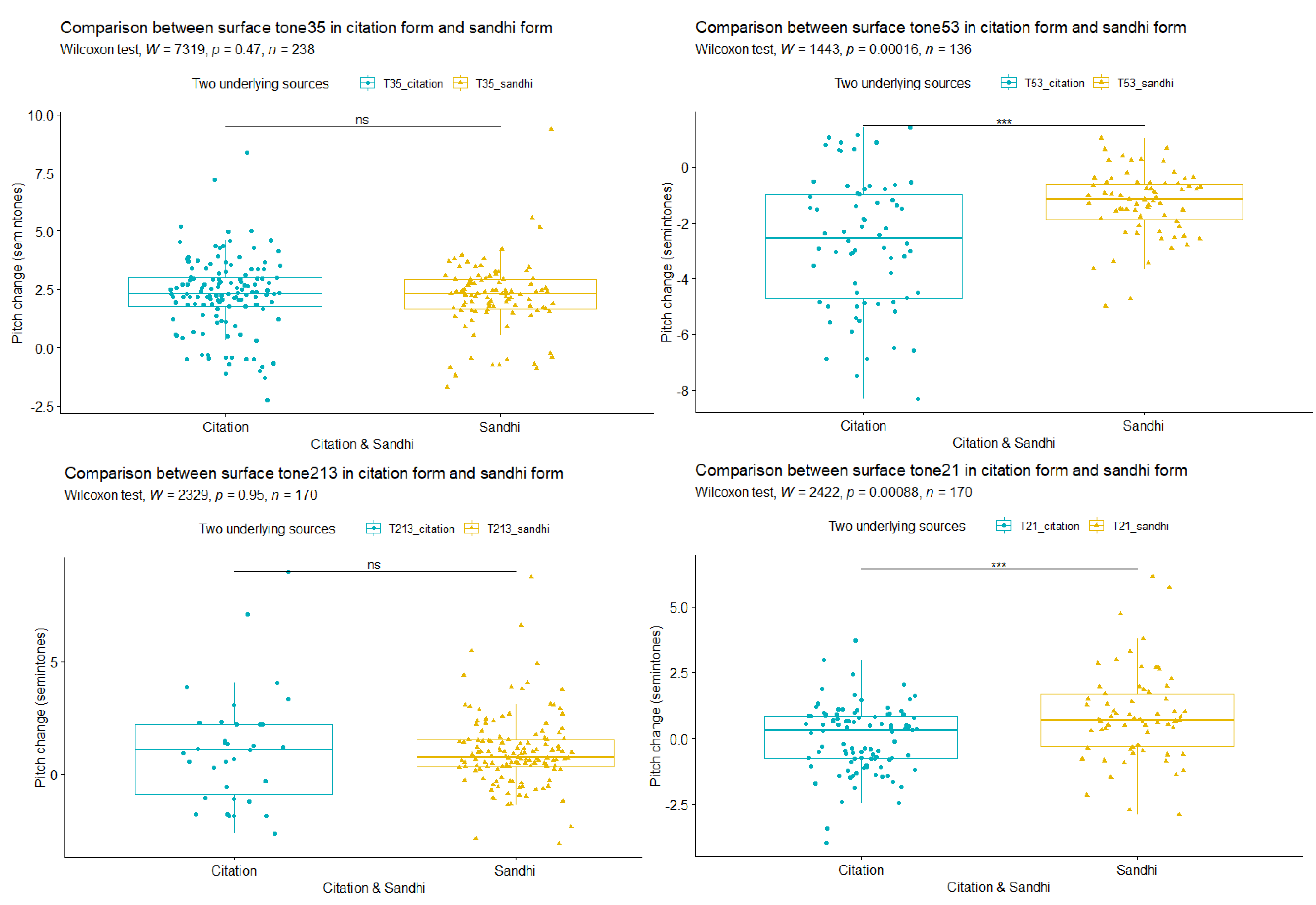

Figure 1. Differences between surface tones /35/, /53/, /213/ and /21/ in citation form and in sandhi form.

\section{Acknowledgements}

The authors wish to acknowledge Sarah Hawkins and Bob Ladd for their suggestions on data analysis and to thank Hai Hua Huang for her enormous help with recruiting participants in Chaozhou. 